\title{
A adesão ao autocuidado de idosos com diabetes mellitus tipo 2: revisão integrativa
}

\author{
Adherence to self-care for elderly people with type 2 diabetes mellitus: integrative review \\ Adhesión al autocuidado de ancianos con diabetes mellitus tipo 2: revisión integradora
}

Diorges Boone da Silva ${ }^{1 *}$, Anderson Lima Cordeiro da Silva ${ }^{1}$, Marinara de Mendonça Bezerra ${ }^{1}$, Fábio Carmona de Jesus Maués².

\section{RESUMO}

Objetivo: Descrever a adesão ao autocuidado em idosos com diabetes mellitus tipo 2. Métodos: Estudo de revisão integrativa, com abordagem qualitativa. A pesquisa bibliográfica foi concretizada em bases de dados, cientificamente válidas, por meio da Biblioteca Virtual em Saúde (BVS), selecionando artigos através dos Descritores em Saúde (DeCS): Diabetes Mellitus; Idosos; Autocuidado, publicados entre os anos de 2015 à 2020, considerando os critérios de elegibilidade e inelegibilidade. Resultados: Foram encontrados 527 artigos na Scielo, 99 na Medline e 3.248 na Lilacs, totalizando 3.874 achados, destes 20 artigos atenderam os critérios elencados para os resultados. A literatura demonstra riqueza quanto aos estudos voltados ao autocuidado, mas existe repetição dos assuntos elencados nos estudos, não tendo um fator que conduza de forma conclusiva a melhora de tal problemática. Considerações finais: Analisar o entendimento dos idosos através das literaturas, e do que tem sido feito para uma promoção de saúde mais efetiva mostrou-se essencial para que fosse possível reconhecer as contribuições dessa estratégia para a melhoria do autocuidado em idosos com diabetes.

Palavras-chave: Diabetes mellitus, Idosos, Autocuidado.

\begin{abstract}
Objective: To describe the adherence to self-care in elderly people with type 2 diabetes mellitus. Methods: Integrative review study, with qualitative approach. The bibliographic research was carried out in scientifically valid databases through the Virtual Health Library (VHL), selecting articles through the Health Descriptors (DeCS): Diabetes Mellitus; Elderly; Self-care, published between 2015 and 2020, considering the eligibility and ineligibility criteria. Results: 527 articles were found in Scielo, 99 in Medline and 3,248 in Lilacs, totaling 3,874 findings, of these 20 articles met the criteria listed for results. The literature shows richness as to the studies focused on self-care, but there is repetition of the subjects listed in the studies, not having a factor that leads conclusively to the improvement of such problem. Final considerations: To analyze the understanding of the elderly through the literature, and of what has been done for a more effective health promotion was essential to be able to recognize the contributions of this strategy to the improvement of self-care in elderly with diabetes.
\end{abstract}

Keywords: Diabetes mellitus, Elderly, Self-care.

\section{RESUMEN}

Objetivo: Describir la adherencia al autocuidado en personas mayores con diabetes mellitus tipo 2. Métodos: Estudio de revisión integradora, con enfoque cualitativo. La investigación bibliográfica se llevó a cabo en bases de datos científicamente válidas a través de la Biblioteca Virtual de Salud (BVS), seleccionando artículos a

${ }^{1}$ Centro Universitário Fametro (CEUNI-FAMETRO), Manaus - AM. *E-mail: diorges248@gmail.com

2Universidade Federal do Amazonas (UFAM), Manaus - AM. 
través de los Descriptores de Salud (DeCS): Diabetes Mellitus; Ancianos; Autocuidado, publicados entre 2015 y 2020, considerando los criterios de elegibilidad y no elegibilidad. Resultados: Se encontraron 527 artículos en Scielo, 99 en Medline y 3.248 en Lilacs, con un total de 3.874 hallazgos, de los cuales 20 artículos cumplieron los criterios de los resultados. La literatura muestra riqueza en cuanto a los estudios enfocados al autocuidado, pero hay repetición de los temas listados en los estudios, no teniendo un factor que conduzca de manera concluyente a la mejora de dicho problema. Consideraciones finales: Analizar la comprensión de los ancianos a través de la literatura, y de lo que se ha hecho para una promoción de la salud más efectiva fue esencial para poder reconocer las contribuciones de esta estrategia a la mejora del autocuidado en los ancianos con diabetes.

Palabras clave: Diabetes mellitus, Ancianos, Autocuidado.

\section{INTRODUÇÃO}

Diabetes mellitus tipo 2 (DM2) caracteriza-se como uma doença complexa atribuída a um conjunto de distúrbios metabólicos de elevados índices hiperglicêmicos, sendo estes causados por defeitos na ação ou na secreção de insulina. O DM2 é o mais predominante dentre os outros tipos de diabetes mellitus (DM) que corresponde de 90 a $95 \%$ dos casos diagnosticados. Seu surgimento se dá principalmente em adultos, resultante da produção insuficiente ou resistência insulínica $(\mathrm{Rl})$, as causas principais estão relacionadas à obesidade e estilo de vida sedentário. Este advento está intimamente ligado ao envelhecimento (SBD, 2018).

O DM está entre uma das doenças crônicas não transmissíveis (DCNT) mais prevalentes, por ter um aumento significativo em todos os países e seu grande índice de morbimortalidade, em particular nas pessoas com idade mais avançada. A maior incidência de DM2 em idosos está diretamente associada a fatores fisiológicos devido às mudanças sistêmicas, as células beta-pancreática continuam a produção insulínica, sendo ineficazes, devido ao organismo desenvolver RI (PRADO MAMB, et al., 2016).

Segundo os dados da International Diabetes Federation (IDF) (2017), estima-se que a nível mundial exista 425 milhões de portadores de diabetes no mundo, tendo como faixa etária pessoas de 20 a 79 anos. A promoção de medidas preventivas para os devidos cuidados pode minimizar os riscos. Os dados da Organização Mundial de Saúde (OMS), apontam que o Brasil está na $4^{\circ}$ posição de maior prevalência, com 16 milhões de pessoas diagnosticadas, ocupando ainda o 5 lugar com indivíduos acima de 65 anos (OMS, 2018).

De acordo com Silva AB (2016), há uma previsão para que até o ano de 2030 o Brasil possa ter aproximadamente 11,3 milhões de pessoas com diabetes, destes cerca de $33 \%$ tenham entre 60 e 79 anos. Em virtude desse cenário, fica claro que promover saúde é essencial para que o Autocuidado (AC) se torne eficaz. Assim, a Atenção Primária em Saúde (APS) intervém diretamente na melhora da qualidade de vida dos idosos. Corroborando, Rodrigues RC (2018), descreveu que fatores como a Depressão; Humor deprimido; Perda de energia e Concentração são desencadeadores para redução ao cuidado.

As particularidades de uma pessoa idosa, evidenciam um cuidado deficitário, pois estão mais propensas a apresentarem doenças crônicas, fragilidades, mais custos, menos recursos sociais e financeiros. Mesmo em situações antagônicas, o idoso deve ter um cuidado estruturado de forma equânime do que é realizado para o adulto, pois envelhecer, ainda que sem doenças, envolve alguma perda funcional (VERAS RC, 2016).

Deste modo, a atuação profissional na educação em saúde se torna um fator importante que traz uma maior eficácia sobre as práticas do AC, possibilitando que o paciente seja também autor nesse processo de medidas de prevenção. Implementar ações na estratégia de saúde, faz com que os pacientes se sintam mais motivados e instruídos a manter uma rotina saudável, tanto como inclui-los nos programas de saúde disponíveis, proporciona melhorias no seu prognóstico (IQUIZE RCC, et al., 2017).

De acordo com Papaléo MP (2002), o impacto socioeconômico na vida de uma pessoa idosa traz grandes transformações, visto que os custos financeiros aumentam, consultas médicas, medicamentos, alimentação saudável, assim os recursos para uma adequada adesão ao tratamento se tornam mais difíceis. 
O Ministério da Saúde (MS) elucida que, para uma melhor aderência as terapêuticas se torna fundamental que a equipe de saúde possa produzir vínculos positivos e propostas pactuadas, assim como, a utilização da avaliação das Atividades de Vida Diária (AVD) que indicam a incapacidade e comprometimento dos idosos (BRASIL, 2006).

Os profissionais de saúde devem estabelecer metodologias ativas direcionadas ao idoso, que possam despertar essas novas responsabilidades que serão trabalhadas em conjunto, visando o atendimento integral ao usuário com DM2. Essas medidas geram um impacto positivo para o paciente devido a transferência de conhecimento que possibilita mudanças para sua própria promoção de saúde (SOUZA VP e VASCONCELOS EMR, 2017).

O estudo de Reberte LM, et al. (2012), apontam que o Sistema Único de Saúde (SUS) é um dos maiores usuários de materiais educativos como instrumento de apoio, sendo estes: Manuais de cuidado em saúde, folhetos e cartilhas. Esses são capazes de promover resultados expressivos. Echer IC (2005), contribui que, a criação destes meios educativos facilita as orientações a serem realizadas, melhorando o trabalho da equipe multidisciplinar na instrução de pacientes e familiares no processo de tratamento, recuperação e AC.

Nessa perspectiva, o educador em saúde deve ficar ciente de que o portador dessa condição crônica compreendeu a importância do AC para um tratamento efetivo em todos os ângulos, desde o controle da hiperglicemia até o cuidado com os pés (DIAS EG, et al., 2017).

O SUS preconiza que a pessoa com DM, tenha acompanhamento multiprofissional por meio da Equipe de Saúde da Família (ESF) na atenção primária. Todos os profissionais envolvidos na ESF possuem atribuições nesse processo, configurando-se uma educação efetiva possibilitando a autonomia. Desta forma, é importante que sua formação atue de maneira generalizada, conduzindo adequadamente um tratamento dentro de cada necessidade (COÊLHO MCVS, et al., 2018).

Visto que o AC pelos portadores de DM2 é de extrema necessidade, envolvendo mudanças comportamentais, além de grandes dificuldades de aceitação, torna-se importante que a tríade, enfermeiro, indivíduo e família, atuem em conjunto para uma melhor adesão as terapêuticas. Portanto, é necessário que a equipe multidisciplinar atribua em suas abordagens um diálogo que possibilite a construção de um plano favorável para que a família e o paciente possam seguir, sempre havendo troca de conhecimento e informação, valorizando sempre o diálogo do idoso, objetivando descrever a adesão ao autocuidado em idosos com diabetes mellitus tipo 2.

\section{MÉTODOS}

Trata-se de um estudo bibliográfico descritivo, com abordagem qualitativa. Optou-se pela escolha da revisão integrativa da literatura, por ser descrita como uma abordagem metodológica mais ampla permitindo a compreensão de um fenômeno analisado através de estudos experimentais e não experimentais, podendo utilizar também dados da literatura teórica e empírica (GIL AC, 2002).

A pesquisa bibliográfica foi concretizada em bases de dados bibliográficas nacionais e internacionais, cientificamente válidas, por meio da Biblioteca Virtual em Saúde (BVS), selecionando artigos através dos Descritores em Saúde (DeCS): Diabetes Mellitus; Idosos; Autocuidado, sendo estes publicados entre os anos de 2015 à 2020, utilizando a expressão " $A N D$ " para efetuar a busca, filtrando idiomas da língua portuguesa e inglesa, disponíveis na Biblioteca Científica Eletrônica Online (SciELO), Literatura Latino-Americano e do Caribe (LILACS) e Medical Literature Analysis and Retrieval System Online (MEDLINE).

No processo de seleção dos artigos, considerou-se aqueles que correspondiam aos critérios de elegibilidade definido: Artigos com data de publicação superiores a 2015; Publicações em idiomas na língua português e inglesa; Artigos que contextualizem a ideia da proposta, bem como os DeCS nas buscas realizadas.

A inelegibilidade foi definida a partir de: Artigos repetidos em demais bases de busca; não se referirem a adultos; artigos que fujam da temática central da pesquisa; estudos sobre outras patologias e ausência de acesso ao texto na íntegra. Os estudos foram sendo considerados elegíveis de acordo com seu 
enquadramento nos critérios, seguidos de avaliação por meio do título, resumo, leitura na integra e desta forma sendo selecionados para a revisão. A triagem foi realizada pelos integrantes da pesquisa, desta forma chegando ao resultado e desenho do estudo, onde todos realizaram o mesmo processo de inclusão e exclusão dos estudos encontrados nas bases de dados.

Os dados extraídos dos artigos, foram discutidos e incluídos em uma tabela para viabilizar a discussão do trabalho, bem como a tabela recebeu os títulos dos artigos, autores, ano de publicação, idioma, plataforma encontrado e uma breve resultado das partes mais relevantes de acordo com a temática proposta.

Através dos DeCS e os critérios citados para busca e seleção, foram encontrados 527 artigos na Scielo, 99 na Medline e 3.248 na Lilacs, totalizando 3.874 achados, destes 20 artigos atenderam os critérios para elencados para os resultados (Quadro 2).

\section{RESULTADOS}

A DM é um tipo DCNT mais frequente no mundo, sendo a quarta principal causa de morte, que consiste em um distúrbio metabólico caracterizada por hiperglicemia persistente, decorrente de deficiência na produção insulínica, resultando em complicações a longo prazo.

Insulina é um hormônio produzido especificamente nas células beta $(\beta)$ das ilhotas de langerhans do pâncreas, hormônio este responsável pela manutenção dos valores adequados de glicose na corrente sanguínea, que atua no transporte do meio intravascular para o intracelular (MONTEIRO MDS e SOUZA SP, 2017).

Quadro 1 - Classificação quanto aos tipos de diabetes.

\begin{tabular}{|c|c|}
\hline Tipos & Descrição \\
\hline DM1 & $\begin{array}{c}\text { Habitualmente a DM1 é diagnosticada na infância ou adolescência e } \\
\text { geralmente corresponde de 5 a 10\% dos casos, sendo este autoimune. }\end{array}$ \\
\hline DM2 & $\begin{array}{c}\text { Acomete indivíduos a partir dos quarenta anos de idade, podendo ser } \\
\text { descrito em faixas etárias inferiores, corresponde de } 90 \text { a 95\% de todos } \\
\text { os casos. Trata-se de uma doença poligênica, herança familiar, } \\
\text { multifatorial, envolve componentes genéticos e ambientais. }\end{array}$ \\
\hline Gestacional & $\begin{array}{c}\text { Estado de hiperglicemia, podendo ser diagnosticados entre o segundo ou } \\
\text { terceiro trimestre gestacional, geralmente se resolve no período pós-parto } \\
\text { e pode frequentemente retornar anos depois. }\end{array}$ \\
\hline
\end{tabular}

Fonte: Silva DB, et al., 2020. Baseado em SBD, 2018.

Os estudos aos quais tivemos acesso, em relação a adesão ao AC de idosos com DM2, foram bem diversificados, apresentando vários aspectos, tipos e formas de cuidados, bem como diferentes tipos de intervenção para uma educação em saúde voltada ao público idoso, contudo ainda deficitário os programas de apoio ao AC, visto que a atenção básica nem sempre dispõem de profissionais o suficiente para promover uma educação efetiva e averiguação da aplicabilidade no dia a dia dos cuidados repassados.

Deste modo, se tornou mais significativo compreender as intervenções por parte da equipe de saúde e a aquisição do AC por parte dos idosos. Ainda assim, as pesquisas encontradas demonstram fragilidade quanto a qualidade das formas de intervenção. Nota-se que, há muito que ser revisto para que conclusões possam ser tomadas frente ao quadro atual do cuidado diário dos idosos.

A literatura demonstra riqueza quanto aos estudos voltados ao $\mathrm{AC}$ em idosos, mas existe repetição dos assuntos elencados nos estudos, não tendo um fator que conduza o que possa ser conclusivo para a melhora de tal problemática enfrentada, havendo muitas demonstrações da gestão, das intervenções das dificuldade dos idosos em atribuir o AC. Todavia não há um programa que de fato melhore o padrão de busca por estes idosos que não dispõem de recursos o suficiente. Os artigos encontrados de acordo com os DeSC, estão dispostos no Quadro 2. 
Quadro 2 - Realização da busca de artigos através dos DeCS e seleção para os resultados e discussão.

\begin{tabular}{|c|c|c|c|c|c|c|}
\hline Base de Dados & DeCS & $\begin{array}{c}\text { Número de } \\
\text { Referências } \\
\text { obtidas }\end{array}$ & $\begin{array}{c}\text { Seleção através } \\
\text { dos critérios de } \\
\text { elegibilidade e } \\
\text { inelegibilidade }\end{array}$ & $\begin{array}{c}\text { Resumos } \\
\text { Analisados } \\
\text { por meio } \\
\text { dos Títulos }\end{array}$ & $\begin{array}{c}\text { Analisados } \\
\text { na integra }\end{array}$ & $\begin{array}{c}\text { Selecionados } \\
\text { para Revisão }\end{array}$ \\
\hline \multirow{2}{*}{ SciELO } & $\begin{array}{c}\text { Diabetes } \\
\text { Mellitus and } \\
\text { Idosos }\end{array}$ & 284 & 81 & 15 & 8 & 5 \\
\cline { 2 - 7 } & $\begin{array}{c}\text { Diabetes and } \\
\text { Autocuidado }\end{array}$ & 243 & 95 & 24 & 13 & 6 \\
\hline \multirow{2}{*}{$\begin{array}{c}\text { Diabetes } \\
\text { Lellitus and } \\
\text { Idosos }\end{array}$} & 63 & 27 & 8 & 6 & 2 \\
\cline { 2 - 7 } & $\begin{array}{c}\text { Diabetes and } \\
\text { Autocuidado }\end{array}$ & 33 & 13 & 5 & 3 & 1 \\
\hline $\begin{array}{c}\text { Diabetes } \\
\text { Mellitus and } \\
\text { Idosos }\end{array}$ & 1.804 & 75 & 9 & 7 & 5 \\
\cline { 2 - 7 } & $\begin{array}{c}\text { Diabetes and } \\
\text { Autocuidado }\end{array}$ & 7.534 & 1.444 & 75 & 6 & 1 \\
\hline
\end{tabular}

Fonte: Silva DB, et al., 2020.

\section{DISCUSSÃO}

Este estudo aborda uma descrição interpretativa dos achados na literatura, integrando a revisão de 20 artigos selecionados, sendo estes voltados para o AC em idosos com DM2, visando propor conhecimento sobre a temática, na qual aborde as dificuldades de se atribuir uma qualidade de vida perante a patologia e 0 AC. No Quadro 3, estão dispostos os resultados elegidos para a discussão do trabalho, visto que esta seleção se deu por meio de alguns fatores para o alcance final da proposta que será aqui realizada.

A Sociedade Brasileira de Diabetes (SBD) (2018), denomina o processo de educação em diabetes, como o desenvolvimento de habilidades que incorporam métodos para que o objetivo seja estabelecido em todas as etapas do tratamento, possibilitando o alcance do AC. Este processo de educação busca atingir pacientes que são diagnosticados com DM bem como seus familiares. Para contribuir de forma positiva com a pessoa idosa, é necessário um maior tempo de educação, conscientização, ouvir as queixas e as dificuldades impostas pelo cliente.

De acordo com Freitas EV e Py L (2017), o envelhecimento é um processo que progride com o tempo, podendo ocorrer declínio funcional dos órgãos, levando a perda de reservas do organismo. As ocorrências destas modificações são predisponentes para a prevalência de DM2. A diminuição da massa magra que também é responsável pela distribuição da glicose mediada pela insulina e o excesso da gordura visceral que aumenta a Resistência Insulínica. Por tanto, fisiologicamente o idoso torna-se mais susceptível ao surgimento de outras doenças.

Diante dessas potencialidades, a assistência de uma equipe multiprofissional se torna essencial na prestação dos cuidados e atividades educativas, contribuindo de maneira significativa na adesão ao tratamento. Proporcionar uma boa qualidade para os idosos faz com eles se sintam assistidos em todas as suas esferas, visando tanto a melhora deste quadro clínico quanto a diminuição de gastos para o sistema público quanto para paciente (MENEZES LTG, et al., 2016).

Pode-se observar que, mesmo havendo compreensão quanto a importância dos horários prescritos das medicações, muitos idosos iniciam o uso com certa desconfiança, mantendo-se alertas a qualquer possível reação adversa ou resposta corporal provocados pelas medicações, grande parte dos idosos não seguem os horários de forma rígida, contudo se orientam pelas principais refeições, sendo está uma forma também de educação em saúde que pode funcionar de forma eficiente para uma conduta de aprazamento dos medicamentos a serem utilizados pelos pacientes diabéticos (SOUZA AS, et al., 2019).

A saúde do idoso é uma integração resultante de fatores decorridos ao longo dos anos, assim como: contribuição familiar; da sociedade e dos profissionais aumentam o viés de autoconfiança, mostrando as potencialidades e o valor que as pessoas idosas possuem, pois parte das grandes dificuldades e abandono de tratamento deste grupo relaciona-se a uma cultura que o desvaloriza (UFSC, 2013). 


\section{Revista Eletrônica Acervo Saúde / Electronic Journal Collection Health | ISSN 2178-2091}

Quadro 3 - Característica dos artigos escolhidos para a revisão integrativa.

\begin{tabular}{|c|c|c|c|c|c|c|}
\hline $\mathbf{N}$ & Título & Autor & Ano & Idioma & Plataforma & Resultados \\
\hline 1 & $\begin{array}{l}\text { Programa de educação em saúde melhora } \\
\text { indicadores de autocuidado em diabetes e } \\
\text { hipertensão. }\end{array}$ & Magri S, et al. & 2020 & Inglês & Lilacs & $\begin{array}{c}\text { Instituir programas, para melhorar o } \\
\text { conhecimento e autocuidado de portadores de } \\
\text { DM, reduz o adoecimento por parte da } \\
\text { patologia. }\end{array}$ \\
\hline 2 & $\begin{array}{l}\text { Caracterização de idosos diabéticos e } \\
\text { fatores associados a adesão terapêutica na } \\
\text { atenção básica. }\end{array}$ & Bastos RAA, et al. & 2018 & Port. & Lilacs & $\begin{array}{l}\text { O nível de escolaridade influência da aderência } \\
\text { ao tratamento em casa, dificuldade de } \\
\text { compreender prescrições e instruções. }\end{array}$ \\
\hline 3 & $\begin{array}{c}\text { Atividade física de lazer em idosos com } \\
\text { diabetes tipo 2: estudo de base } \\
\text { populacional }\end{array}$ & Lima AP, Cardoso FB. & 2019 & Port. & Lilacs & $\begin{array}{l}\text { Os autores inferem que, } 77 \% \text { dos idosos com } \\
\text { DM2 estão inativos fisicamente. }\end{array}$ \\
\hline 4 & $\begin{array}{c}\text { Adesão ao tratamento de diabetes mellitus } \\
\text { e relação com a assistência na atenção } \\
\text { primária }\end{array}$ & Santos AL, et al. & 2020 & Port. & Lilacs & $\begin{array}{l}\text { O ambiente estrutural é um dos fatores que } \\
\text { influenciam para uma adesão positiva do } \\
\text { tratamento. }\end{array}$ \\
\hline 5 & $\begin{array}{l}\text { Avaliação da educação em grupo de } \\
\text { diabetes na atenção primária à saúde }\end{array}$ & Torres HC, et al. & 2016 & Port. & Lilacs & $\begin{array}{c}\text { O conhecimento em relação a doença, se torna } \\
\text { decisivo na compreensão acerca do } \\
\text { autocuidado. }\end{array}$ \\
\hline 6 & $\begin{array}{l}\text { Resiliência e autocuidado de pessoas } \\
\text { idosas com diabetes mellitus }\end{array}$ & Vicente MC, et al. & 2019 & Port. & Lilacs & $\begin{array}{l}\text { Existem grandes limitações associadas aso } \\
\text { aspectos do autocuidado. }\end{array}$ \\
\hline 7 & $\begin{array}{l}\text { Validade e concordância do diabetes } \\
\text { mellitus referido em idosos }\end{array}$ & $\begin{array}{c}\text { Menezes TN, Oliveira } \\
\text { ECT. }\end{array}$ & 2019 & Inglês & Medline & $\begin{array}{l}\text { Promover recursos que viabilizem a } \\
\text { transformação de hábitos diários. }\end{array}$ \\
\hline 8 & $\begin{array}{l}\text { Alfabetização em saúde e autocuidado em } \\
\text { pessoas com diabetes mellitus tipo } 2\end{array}$ & Moura NS, et al. & 2019 & Inglês & Medline & $\begin{array}{c}\text { O letramento funcional em saúde, tem sido um } \\
\text { dos fatores que predispõem uma baixa } \\
\text { aquisição do autocuidado. }\end{array}$ \\
\hline 9 & $\begin{array}{c}\text { Diabetes mellitus referida: incidência e } \\
\text { determinantes, em coorte de idosos do } \\
\text { município de São Paulo, Brasil, Estudo } \\
\text { SABE - Saúde, Bem-Estar e } \\
\text { Envelhecimento }\end{array}$ & Roediger ME, et al. & 2018 & Inglês & Medline & $\begin{array}{l}\text { Escassez de determinantes aos idosos no } \\
\text { Brasil, para reduzir está incidência. }\end{array}$ \\
\hline 10 & $\begin{array}{c}\text { Conhecimento sobre o diabetes e atitude } \\
\text { para o autocuidado de idosos na atenção } \\
\text { primária à saúde }\end{array}$ & Borba AKOT, et al. & 2019 & Port. & Scielo & $\begin{array}{l}\text { Homens com diabetes, tem maiores resistência } \\
\text { de praticar o autocuidado. }\end{array}$ \\
\hline
\end{tabular}




\section{Revista Eletrônica Acervo Saúde / Electronic Journal Collection Health | ISSN 2178-2091}

\begin{tabular}{|c|c|c|c|c|c|c|}
\hline $\mathbf{N}$ & Título & Autor & Ano & Idioma & Plataforma & Resultados \\
\hline 11 & $\begin{array}{c}\text { Construção, validação e adequação cultural } \\
\text { do protocolo COMPASSO: Adesão ao } \\
\text { autocuidado em diabetes }\end{array}$ & Fernandes BSM, et al. & 2016 & Port. & Scielo & $\begin{array}{c}\text { Conhecer o contexto de vida aproxima a } \\
\text { prática clínica do profissional para atribuir a } \\
\text { autocuidado. }\end{array}$ \\
\hline 12 & $\begin{array}{l}\text { Adesão e empoderamento de usuários com } \\
\text { diabetes mellitus para práticas de } \\
\text { autocuidado: ensaio clínico randomizado }\end{array}$ & Macedo MML, et al. & 2017 & Port. & Scielo & $\begin{array}{c}\text { A prática educativa em grupo e o } \\
\text { empoderamento, constrói novos } \\
\text { conhecimentos e participação ativa, assim } \\
\text { como protocolos para mudanças diárias. }\end{array}$ \\
\hline 13 & $\begin{array}{c}\text { Intervenção educativa para a promoção do } \\
\text { autocuidado de idosos com diabetes } \\
\text { mellitus }\end{array}$ & Marques MB, et al. & 2019 & Port. & Scielo & $\begin{array}{c}\text { Atividades desenvolvidas por enfermeiros, } \\
\text { direcionadas para os idosos visando a } \\
\text { prevenção de complicações por parte do } \\
\text { diabetes. }\end{array}$ \\
\hline 14 & $\begin{array}{l}\text { Experiências, necessidades e expectativas } \\
\text { de pessoas com diabetes mellitus. }\end{array}$ & Silva JA, et al. & 2018 & Port. & Scielo & $\begin{array}{l}\text { A qualidade de vida, está entre o antes e após } \\
\text { o diagnóstico, exigindo mudanças de hábitos } \\
\text { de vida, nem sempre havendo negociação. }\end{array}$ \\
\hline 15 & $\begin{array}{c}\text { Avaliação da visita domiciliar para o } \\
\text { empoderamento do autocuidado em } \\
\text { diabetes }\end{array}$ & Sousa DAS, et al. & 2017 & Port. & Scielo & $\begin{array}{l}\text { As visitas domiciliares contribuem para a } \\
\text { adesão ao autocuidado. }\end{array}$ \\
\hline 16 & $\begin{array}{c}\text { Intervenções para a aquisição do } \\
\text { autocuidado terapêutico da pessoa com } \\
\text { diabetes mellitus: revisão sistemática da } \\
\text { literatura }\end{array}$ & Cardoso AF, et al. & 2015 & Inglês & Scielo & $\begin{array}{c}\text { A educação promovida se torna positiva para o } \\
\text { autocuidado, contudo a mesma deve ser } \\
\text { mantida para que seja eficaz. }\end{array}$ \\
\hline 17 & $\begin{array}{c}\text { Atividades de autocuidado e suas relações } \\
\text { com controle metabólico e clínico das } \\
\text { pessoas com diabetes mellitus }\end{array}$ & Coelho ACM, et al. & 2015 & Inglês & Scielo & $\begin{array}{c}\text { Existe compreensão, quando a dieta e fatores } \\
\text { que influenciam para uma vida saudável, } \\
\text { contudo acabam não seguindo. }\end{array}$ \\
\hline 18 & $\begin{array}{c}\text { Short Message Service (SMS) e promoção } \\
\text { do autocuidado em DM2: revisão } \\
\text { integrativa }\end{array}$ & Hovadick AC, et al. & 2019 & Inglês & Scielo & $\begin{array}{l}\text { Uso da tecnologia para instruir pessoas quanto } \\
\text { ao seu autocuidado de forma a motivar e } \\
\text { fortalecer suas atividades diárias. }\end{array}$ \\
\hline 19 & $\begin{array}{l}\text { Qualidade de vida e o tempo do } \\
\text { diagnóstico do diabetes mellitus em idosos }\end{array}$ & Lima LR, et al. & 2018 & Inglês & Scielo & $\begin{array}{l}\text { As relações sociais realizadas pelos } \\
\text { enfermeiros, são referidas como um apoio para } \\
\text { os idosos como positivas. }\end{array}$ \\
\hline 20 & $\begin{array}{c}\text { Atividades de autocuidado, variáveis } \\
\text { sociodemográficas, tratamento e sintomas } \\
\text { depressivos entre idosos com Diabetes } \\
\text { Mellitus }\end{array}$ & Trevizani FA, et al. & 2019 & Inglês & Scielo & $\begin{array}{c}\text { Idosos sem um parceiro (a) tem maiores } \\
\text { dificuldades, assim como a renda é outro fator } \\
\text { que influencia na adesão. }\end{array}$ \\
\hline
\end{tabular}

Fonte: Silva DB, et al., 2020. 
Os autores Silva JA, et al. (2018); Lima AP e Cardoso FB (2019); inferem que, 77\% dos idosos com DM2 estão inativos fisicamente, sabendo que o envelhecimento vem acompanhado de várias modificações, como a vulnerabilidade, diminuição de sua capacidade, assim a pessoa idosa com diabetes enfrenta maiores dificuldades para gerenciar o AC no dia-a-dia, comprometendo sua qualidade de vida.

Os problemas associados ao diabetes podem ser evitados, desde que a adoção de medidas e cuidados especializados possa ser atribuída de forma regular, tendo em vista que os pacientes precisam previamente de conhecimentos sobre a patologia e as práticas de cuidado, cabendo ao profissional de saúde fornecer praticas educativas em grupo visando a prevenção de morbidades associadas a patologia, por tanto, conhecer o contexto de vida aproxima a prática clínica do profissional (TORRES HC, et al., 2016; FERNANDES BSM, et al., 2016; MACEDO MML, et al., 2017; MARQUES MB, et al., 2019).

Para a teórica Dorothea Elizabeth Orem, o $A C$ é a prática de atividades que os indivíduos iniciam pessoalmente e desempenham em seu próprio benefício para manter a vida, a saúde e o bem-estar. Este podendo concentrar-se no profissional juntamente com o paciente, na tentativa de se identificar os déficits da capacidade individual, procurando medidas que potencializem as habilidades que o cliente possui para cuidar de si, podendo assim fortalecer seu conhecimento e suas dificuldades (JULIA BG, 2000; OREM DO, 1971).

Salienta-se então que, prover medidas que construam um cuidado eficaz, instruindo o AC para população idosa, torna-se uma prevenção primária, evitando diversas comorbidades que afetam de forma prejudicial o envelhecimento. Podemos elucidar que, o AC é visto também como o indivíduo se encontra, se autoanalisa, seus aspectos físicos, sociodemográficos, psicológicos e condições familiares.

A atribuição da tecnologia para instruir pessoas quanto ao seu AC, contribuiria com as relações sociais realizadas pelos profissionais da enfermagem, sendo estas referidas como um apoio positivo para os idosos. Contudo, toda relação, tipo de educação e programas que sejam efetivos devem manter-se ativos para que sejam eficazes reduzindo o adoecimento por conta da patologia, para que o processo do AC não perca o significado por parte deste público que requer uma atenção equânime (CARDOSO AF, et al., 2015; LIMA LR, et al., 2018; HOVADICK AC, et al., 2019; MAGRI S, et al., 2020).

Ao optar-se por uma terapia medicamentosa, é importante orientar para as mudanças no estilo de vida, visto que os resultados positivos para uma patologia não advêm apenas dos efeitos das drogas, mas em associação com outros fatores. A prescrição de um agente antidiabético seguirá alguns aspectos, como: mecanismo de Resistência Insulínica; progressão da falência da célula $\beta$, transtornos metabólicos, e repercussões micro e macrovasculares. Os Antidiabéticos orais reduzem os níveis glicêmicos, objetivando a normalidade (em jejum $<100 \mathrm{mg} / \mathrm{dL}$ e pós-prandial $<140 \mathrm{mg} / \mathrm{dL}$ ). Quando não tratada, os sintomas podem se agravar, contribuindo para a manifestação de outras doenças, ficando evidente que os cuidados devem ser integrais em conjunto com a equipe de saúde (SILVA AB, et al., 2016).

Deste modo, instituir o encorajamento para que o idoso possa dar seguimento em suas rotinas, despertando um novo olhar para si, onde tanto a família quanto a equipe multiprofissional serão vistas como bastidores deste processo, valorizando-o como ator principal neste processo, fortificando a autoconfiança fazendo assim um gerenciamento de sua vida de forma mais autônoma. A Figura 1 retrata as categorias abordadas pela teoria de Orem no aspecto do AC.

Figura 1 - Categorias estabelecidas por Orem nos requisitos do AC.

\begin{tabular}{|c|c|}
\hline Universal & $\begin{array}{l}\text {-Processos de vida } \\
\text { - Manutenção de integridade } \\
\text {-Atividades de vida diária }\end{array}$ \\
\hline Desenvolvimento & $\begin{array}{l}\text {-Adaptação de um novo trabalho } \\
\text {-Adaptação às modificações do corpo }\end{array}$ \\
\hline Desvio de saúde & $\begin{array}{l}\text { - São exigidos em condições de doença, } \\
\text { médicas exigidas. }\end{array}$ \\
\hline
\end{tabular}

Fonte: Silva DB, et al., 2020. 
Nesta perspectiva relaciona-se métodos de ajuda pra proferir uma forma efetiva que contribua com as necessidades do paciente, como: orientar; proporcionar apoio físico e psicológico e manter um ambiente de apoio ao desenvolvimento pessoal, as visitas domiciliares são citadas como formas de integrar este desenvolvimento de forma universal e oportunizando um contexto de saúde extra hospitalar (SOUSA DAS, et al., 2017).

O estudo de Trevizani FA, et al. (2019), contribui ainda com os fatores que, influenciam para uma autonegligência do AC em idosos, como a ausência de um parceiro (a), esses apresentam maiores dificuldades para cuidarem de si, a masculinidade ainda é um fator que gera resistência em tal prática, assim como a renda é outro fator que inviabiliza a adesão em alguns casos (BORBA AKOT, et al., 2019).

Nesta perspectiva, autores apontam que existe um pouco de entendimento, no que diz respeito a dieta e fatores que influenciam para uma vida saudável, contudo acabam não seguindo, visto que o nível de escolaridade, letramento funcional em saúde, dificuldade de compreender as prescrições e instruções é mencionado por outros estudos como influência para a uma baixa adesão e aquisição do AC (COELHO ACM, et al., 2015; BASTOS RAA, et al., 2018; SANTOS AL, et al., 2020).

A literatura apresenta limitações no que concerne a resolutividade no aspecto do $A C$, visto que a temática é muito aborda nas pesquisa e publicações, existindo uma escassez de determinantes aos idosos no Brasil. Para reduzir esta incidência, é preciso que medidas de promoção de recursos possam viabilizar transformações de hábitos diários, bem como proferir mudanças culturais na forma com que a população trata as questões alimentares e estar constantemente sedentária (ROEDIGER ME, et al., 2018; MENEZES TN e OLIVEIRA ECT, 2019; VICENTE MC, et al., 2019).

\section{CONSIDERAÇÕES FINAIS}

Sabendo-se que a diabetes é uma das principais doenças simultâneas ao envelhecimento, que leva ao surgimento de outras complicações, analisar os artigos sobre este aspecto demonstrou relevância para compreensão das dificuldades do AC por parte da população idosa, assim como demonstra as formas que a equipes de saúde e gestão atuam neste seguimento, no intuito de melhorar a qualidade de vida desta população. Analisar o entendimento dos idosos através das literaturas, e do que tem sido feito para uma promoção de saúde mais efetiva mostrou-se essencial para que fosse possível reconhecer as contribuições dessa estratégia para a melhoria do AC em diabetes. Desse modo, reiterasse-a importância de incentivar práticas educativas, investimentos financeiros, programas dentro dos lares que sejam efetivos e contínuos, fazendo busca ativa e contribuindo de fato com uma melhor qualidade de vida, prezando pela equidade da população idosa com está condição de saúde.

\section{REFERÊNCIAS}

1. BASTOS RAA, et al. Caracterização de idosos diabéticos e fatores associados a adesão terapêutica na atenção básica. Revista Nursing, 2018; 21 (242): 2254- 2259. Licere, Belo Horizonte, 2019; 22(2).

2. BORBA AKOT, et al. Conhecimento sobre o diabetes e atitude para o autocuidado de idosos na atenção primária à saúde. Ciênc. saúde coletiva; 2019; 24(1).

3. CARDOSO AF, et al. Intervenções para a aquisição do autocuidado terapêutico da pessoa com diabetes mellitus: revisão sistemática da literatura. Ver port saúde pública. 2015; 33(2): 246-255.

4. COELHO ACM, et al. Atividades de autocuidado e suas relações com controle metabólico e clínico das pessoas com diabetes mellitus. Texto Contexto Enferm, Florianópolis, 2015; 24(3): 697-705.

5. COÊLHO MCVS, et al. Formação em educação em diabetes: Significados atribuídos por enfermeiros da atenção primária. Revista brasileira de Enfermagem, Educação e Ensino em Enfermagem 2018.

6. DIAS EG, et al. Desafios da prática do autocuidado do idoso portador de diabetes mellitus tipo 2. Revista SUSTINERE, Rio de Janeiro, 2017; 5(1): 38-53.

7. ECHER IC. Elaboração de manuais de orientação para o cuidado em saúde. Rev. Latino-Am. Enfermagem [online]. 2005, 13(5): 754-757.

8. FERNANDES BSM, et al. Construção, validação e adequação cultural do protocolo COMPASSO: Adesão ao autocuidado em diabetes. Acta Paul Enferm. 2016; 29(4):421-9.

9. FREITAS EV e PY L. Tratado de Geriatria e Gerontologia - 4. ed- Rio de Janeiro: Guanabara Koogan, 2017. Capítulo 75, Página 846. 
10. HOVADICK AC, et al. Short Message Service (SMS) e promoção do autocuidado em DM2: revisão integrativa. Acta Paul Enferm. 2019; 32(2):210-9.

11. IQUIZE RCC. Práticas educativas no paciente diabético e perspectiva do profissional de saúde: uma revisão sistemática. Universidade de Brasília - UnB. Brasília, DF, Brasil J Bras Nefrol 2017;39(2):196-204.

12. LIMA AP, CARDOSO FB. Atividade física de lazer em idosos com diabetes tipo 2:estudo de base populacional. Licere, Belo Horizonte, 2019; 22(2).

13. LIMA, RL, et al. Qualidade de vida e o tempo do diagnóstico do diabetes mellitus em idosos. Rev. Bras. Geriatr. Gerontol., Rio de Janeiro, 2018; 21(2): 180-190.

14. MACEDO MML, et al. Adesão e empoderamento de usuários com diabetes mellitus para práticas de autocuidado: ensaio clínico randomizado. Rev Esc Enferm USP. 2017;51:e03278.

15. MAGRI S, et al. Programa de educação em saúde melhora indicadores de autocuidado em diabetes e hipertensão. Reciis - Rev Eletron Comun Inf Inov Saúde. 2020 abr.-jun.;14(2):386-400.

16. MARQUES MB, et al. Intervenção educativa para a promoção do autocuidado de idosos com diabetes mellitus. Rev Esc Enferm USP · 2019;53:e03517.

17. MENEZES LTG, et al. Assistência de Enfermagem ao Idoso com Diabetes Mellitus: Uma revisão de literatura. Congresso Nacional de Envelhecimento Humano 2016.

18. MENEZES TN, OLIVEIRA ECT. Validade e concordância do diabetes mellitus referido em idosos. Ciência \& Saúde Coletiva, 24(1):27-34, 2019.

19. MONTERIO MDS e SOUZA S.P. Autocuidado praticado por idosos com diabetes mellitus em uma unidade básica de saúde - PARINTINS-AM, 2017.

20. MOURA NS, et al. Alfabetização em saúde e autocuidado em pessoas com diabetes mellitus tipo 2. Rev Bras Enferm [Internet]. 2019;72(3):734-40.

21. NETTO MP. O estudo da velhice no século XX: histórico, definição do campo e termos básicos. In: FREITAS, E. V. et al. Tratado de Geriatria e Gerontologia. Rio de Janeiro: Guanabara Koogan, 2002.

22. PRADOMAMB, et al. Diabetes em idosos: uso de medicamentos e risco de interação medicamentosa. Departamento de Saúde Coletiva, Faculdade de Ciências Médicas, Unicamp, Campinas SP, 21(11):3447-3458, 2016.

23. REBERTE LM, et al. Process of construction of an educational booklet for health promotion of pregnant women. Rev. Latino-Am. Enfermagem [online]. 2012, vol.20, n.1, pp.101-108. ISSN 1518-8345.

24. RODRIGUES RC. Dialogando sobre as vivências com diabetes mellitus: subsídio para o cuidado educativo de Enfermagem. REME - Revista Mineira de Enfermagem 2018.

25. ROEDIGER ME, et al. Diabetes mellitus referida: incidência e determinantes, em coorte de idosos do município de São Paulo, Brasil, Estudo SABE - Saúde, Bem-Estar e Envelhecimento. Ciência \& Saúde Coletiva, 23(11):39133922, 2018.

26. SANTOS AL, et al. Adesão ao tratamento de diabetes mellitus e relação com a assistência na atenção primária. REMERev Min Enferm. 2020;24:e-1279.

27. SILVA AB. Prevalência de diabetes mellitus e adesão medicamentosa em idosos da Estratégia Saúde da Família de Porto Alegre/RS. Cad. Saúde Colet., 2016, Rio de Janeiro, 24 (3): 308-316.

28. SILVA JA, et al. Experiências, necessidades e expectativas de pessoas com diabetes mellitus. Rev. bioét. (Impr.). 2018; 26 (1): 95-101

29. SOUSA DAS, et al. Avaliação da visita domiciliar para o empoderamento do autocuidado em diabetes. Acta Paul Enferm. 2017; 30(4):350-7.

30. SOUZA VP e VASCONCELOS EMR. Educação em saúde como estratégia para o controle do diabetes mellitus: Revisão integrativa da literatura. Revista Baiana de Saúde Pública. v. 41, n. 1, p. 177-195 jan./mar. 2017.

31. TORRES HC, et al. Avaliação da educação em grupo de diabetes na atenção primária à saúde. Ciência y Enfermeria xxii (3): 35-45, 2016.

32. TREVIZANI FA, et al. Atividades de autocuidado, variáveis sociodemográficas, tratamento e sintomas depressivos entre idosos com Diabetes Mellitus.Rev Bras Enferm. 2019;72(Suppl 2):27-34.

33. VERAS RO, et al. Care pathway for the elderly: detailing the model. Rev. bras. geriatr. gerontol. [online]. 2016, vol.19, n.6, pp.887-905. ISSN 1981-2256.

34. VICENTE MC, et al. Resiliência e autocuidado de pessoas idosas com diabetes mellitus Rev Rene. 2019;20:e33947. 